\title{
Risk Factors For Recurrence of Peptic Ulcer Disease: A Retrospective Study In Tertiary Care Referral Center
}

\section{Yaser Alsinnari}

King Saud Bin Abdulaziz University for Health Sciences

Mohammed S. Alqarni

King Saud Bin Abdulaziz University for Health Sciences

Meshari Attar

King Saud Bin Abdulaziz University for Health Sciences

Ziad M. Bukhari

King Saud Bin Abdulaziz University for Health Sciences

Faisal Baabbad

King Saud Bin Abdulaziz University for Health Sciences

Mohammed Almutairi

King Saud Bin Abdulaziz University for Health Sciences

Mohammed Hasosah ( $\nabla$ myhasosah@yahoo.com )

King Saud Bin Abdulaziz University for Health Sciences

\section{Research Article}

Keywords: Peptic ulcer disease, recurrence, $\mathrm{H}$ pylori.

Posted Date: January 12th, 2022

DOl: https://doi.org/10.21203/rs.3.rs-1184031/v1

License: (c) (i) This work is licensed under a Creative Commons Attribution 4.0 International License. Read Full License

Version of Record: A version of this preprint was published at Cureus on February 7th, 2022. See the published version at https://doi.org/10.7759/cureus.22001. 


\section{Abstract}

Backgrounds: Peptic ulcer disease (PUD) is a common gastrointestinal tract disease characterized by mucosal damage secondary to pepsin and gastric acid secretion. The aim of this study was to evaluate the five-year recurrence rate for treated patients with PUD and risk factors contributing to PUD relapses.

Methods: From 2016 through 2021, all patients with endoscopy-proved PUD were identified by reviewing medical records (Best-Care system). Possible risk factors including smoking, non-steroidal antiinflammatory drugs (NSAIDs), aspirin, alcohol, caffeine, and steroid were analyzed by univariate analysis. Treatment outcomes, 5-year recurrence rate, and mortality rate were assessed.

Results: Among 223 patients, there were 187 (83.8\%), who diagnosed endoscopy-proved PUD and 36 (16.2\%), who diagnosed clinical PUD. Among them, 126 (56.5\%) patients were males and the mean age was $62 \pm 2$ years. The five years recurrence rate of PUD was $30.9 \%$. There was no significant difference in the recurrence rate between the duodenal ulcer (33.3\%) and the gastric ulcer (28.8\%). By univariate analysis, the use of steroid and NSAID and H. pylori infection were potential risk factors for PUD $(\mathrm{P}<$ 0.005). The common complication of PUD was gastrointestinal bleeding (34.1\%). Patients who had a complicated PUD were associated with a higher rate of recurrence (45.9\%) compared to the uncomplicated PUD (19.2\%) ( $P>0.05)$.

Conclusion: Our findings demonstrated that the five years recurrence rate of PUD was $30.9 \%$. The use of steroid and NSAID and $\mathrm{H}$. pylori infection were risk factors for recurrence of PUD. PUD places a significant burden on health care systems. Therefore, a multicenter prospective study is needed for effective management to prevent recurrence and complications of PUD.

\section{Introduction:}

Peptic ulcers are open sores formed on the inner lining of the stomach and the upper part of the small intestine. Peptic ulcer disease (PUD) is a common pathology in gastroenterology. The incidence rate of PUD ranges from $10 \%$ to $19 \%$ and impact 4 million people globally each year. One of the hallmarks of peptic ulcer symptoms is abdominal pain, which is usually relieved by food intake or antacids [1]. The occurrence of PUD was recorded at about 1.5 percent to 3 percent. Perforated peptic ulcer (PPU) is a severe complication of PUD, and people with PPU also have severe abdomen with a high risk of long-term illness and fatality. [2]. Omeprazole is widely recognized worldwide as the best pharmacological therapy for both gastric and duodenal ulcers of the stomach [3].

Helicobacter pylori is the most common cause of peptic ulcers and the long-term consumption of nonsteroidal anti-inflammatory drugs (NSAIDs) like sodium naproxen and ibuprofen (Advil, Motrin IB, others). Symptoms of peptic ulcer disease are complex and can include stomach pain, weight loss, nausea, vomiting, and bleeding or perforation of serious diseases. [4]. 
Exterminating $\mathrm{H}$. pylori helps treat PUD and significantly decreases its recurrence. Effective elimination of $\mathrm{H}$. pylori is by far the most critical factor influencing the relapse of PUD, even though age, drugs, and the prevalence of chronic diseases also impact its recurrence. The risk of relapse of $\mathrm{H}$. pylori infection disease associated with peptic ulcers in adults can be decreased when the bacterium $\mathrm{H}$. Pylori is successfully eradicated. Although the findings differ from study to study. The five-year recurrence rate of PUD is below 5\%, if there are no potential complications related to NSAIDs, after $\mathrm{H}$. pylori eradication [5].

The consumption of Nonsteroidal anti-inflammatory drugs (NSAIDs) and acetylsalicylic acid (ASA) which leads to adverse gastrointestinal events such as peptic ulcer are becoming more extensive. It is also conceivable that the use of NSAIDs is associated with peptic ulcer complications, such as upper gastrointestinal hemorrhage or perforation. Peptic ulcer disease also has a significant burden on health care costs. The overall cost of peptic ulcer disease in the USA, including both actual expenses and loss of productivity at work, is estimated to be 5.65 billion USD per year [6]. Consumption therapy of (NSAIDs) is well known and is associated with an increased risk of peptic ulcers and non-variceal upper gastrointestinal bleeding. Findings also say that the colon may also be impaired because of excessive use of NSAIDs. Also, the use of these medications is increasing in a growing elderly population. Various studies demonstrated that the use of proton pump inhibitors (PPIs) reduces upper GI harm and the likelihood of upper $\mathrm{Gl}$ complications but is not beneficial in avoiding small intestinal mucosal damage associated with NSAIDs [7]. Chan et al. found that treatment with proton pump inhibitors (PPIs) was more effective in minimizing persistent bleeding over 6 months than $\mathrm{H}$. pylori elimination alone. In clinical settings, it is essential to recommend concomitant PPIs for all users of NSAIDs who have a PUD medical history of complications.

The aim of our study is to evaluate the five-year recurrence rate for patients with treated peptic ulcer disease and factors contributing to relapse. Analyzing PUD complications and their impact along with previous treatment regimen and their effects to determine efficacy of medications used to prevent recurrence

\section{Methods:}

This retrospective cross-sectional study included all peptic ulcer patients diagnosed from 2016 to 2021 at King Abdulaziz Medical City in Jeddah, Saudi Arabia. The inclusion criteria were all patients diagnosed of peptic ulcer disease from 2016 to 2021, and patients who were treated for peptic ulcer during that period. The exclusion criteria were all patients with gastric cancer or comorbidities that affect gastrointestinal system such as inflammatory bowel disease. Both inclusion and exclusion criteria were reviewed on BestCare system which is the medical record system at King Abdulaziz Medical city. This research commenced by reviewing the inclusion and exclusion criteria on the BestCare system and a total of 223 patients were selected for this study. A data sheet was constructed which had demographic variables such as age, gender, comorbidities such as diabetes, hypertension, and psychological history such as stress, anxiety, and post-traumatic stress disorder (PTSD). Other variables from treatment regimen were recorded such as date of diagnosis, type of PUD, proton pump inhibitor (PPI) usage and type of PPI, H2 
antagonist and its type, the use of antibiotics and its type, and cytoprotective agents. As well as diagnostic modality, H. pylori infection, and Zollinger-Ellison syndrome. Risk factors were also recorded in the data sheet which included smoking, NSAID, Aspirin, Alcohol, Caffeine, and Steroid. Treatment outcomes were also collected in the data sheet which included side effects, 5-year recurrence rate, date of recurrence, date of first and last visit, complications and type of complications encountered. Recurrence is noted if PUD reoccurred within 5-years from previously treated PUD which is diagnosed by endoscopy regardless of symptoms. Then all data were collected from the medical record software system BestCare.

The study was in accordance with the Declaration of Helsinki and was ethically approved by the institutional Review Board (IRB) at King Abdullah International Medical Research Center (KAIMARC), IRB reference number IRBC/1992/21 [8].

All data were then entered and analyzed using Statistical Package for the Social Sciences (SPSS) version 23 (IBM corp., Armonk, NY) [9]. Numerical findings and demographic data collected from the data sheet were computed and presented by frequency and percentage. In addition, a data mean was calculated, and all numerical findings were systematically arranged to provide quantitative data summary and descriptive data analysis. Categorical variables are presented as frequencies and percentages and continuous variables as standard deviations or medians; interquartile ranges were used when the distributions were skewed.

Independent variables with $\mathrm{P}$ value of $<0.05$ were considered statistically significant [9].

\section{Results:}

223 patients were included in this study. 126 (56.5\%) patients were males, and 97 (43.5\%) were females. The age of the study population was ranging from 5 to 112 years old with a mean age of $62 \pm 21$. Of the study population, diabetes was recorded in 115 (51.6\%), and hypertension in 136 (61\%). PUD was diagnosed using endoscopy and histology in 187 (83.8\%), and clinical diagnosis in $36(16.2 \%)$ of the patients. The five years recurrence rate of PUD was $30.9 \%$ which was seen in 69 patients. No significant difference in the recurrence rate between the duodenal (33.3\%) and the gastric ulcer (28.8\%). Risk factors were compared against the recurrence rate as shown in Table 1. Various treatment regimens and medication were documented. The association between recurrence and treatment regimen is shown in Table 2. For PPI, the combination of omeprazole and esomeprazole had a rate of recurrence (51.4\%) higher than the use of omeprazole $(27.1 \%)$ or esomeprazole $(29.3 \%)$ alone (P-value $=0.01)$. The medication used in 22 patients on $\mathrm{H} 2$ antagonist therapy was ranitidine. The antibiotic regimen used in the study population is the triple antibiotic H.pylori eradication therapy. 98 (43.9\%) patients had a complicated PUD. Patients who had a complicated PUD were associated with a higher rate of recurrence (45.9\%) compared to the uncomplicated PUD (19.2\%) (P-value > 0.05). Different types of complications were seen as shown in Table 3. Mortality rate among the study population was $31.4 \%$ with no statistically significant association with PUD recurrence. 
Table 1

Risk factors associated with 5-years recurrence of PUD

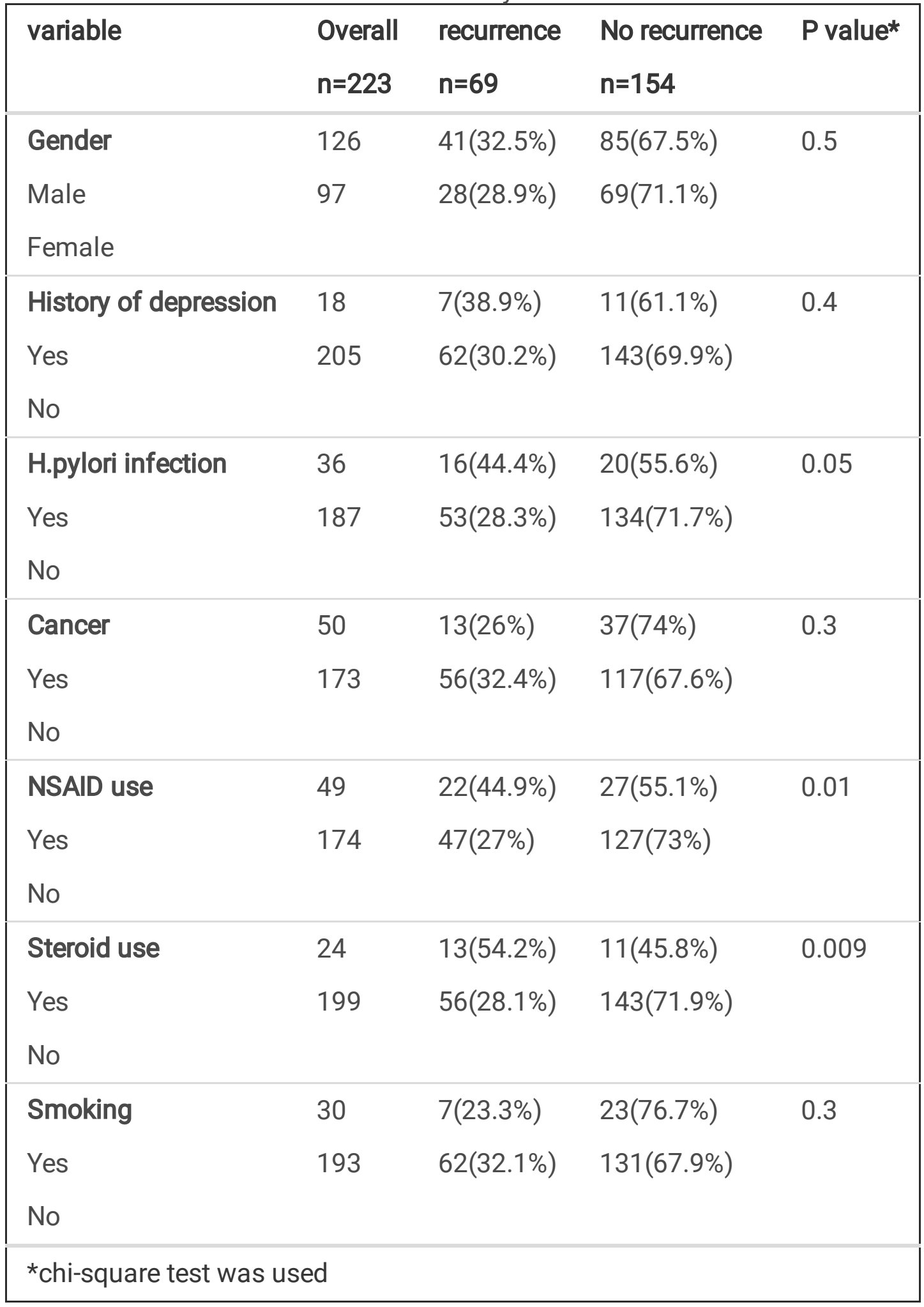


Table 2

Treatments associated with 5-years recurrence of PUD.

\begin{tabular}{|lllll|}
\hline variable & Overall & recurrence & No recurrence & P value* \\
& $\mathbf{n = 2 2 3}$ & $\mathbf{n = 6 9}$ & $\mathbf{n = 1 5 4}$ & \\
\hline Major Treatment plan & 15 & $1(6.7 \%)$ & $14(93.3 \%)$ & 0.02 \\
Avoiding risk factors & 30 & $15(50 \%)$ & $15(50 \%)$ & \\
H.pylori eradication & 145 & $44(30.3 \%)$ & $101(69.7 \%)$ & \\
Acid suppression therapy & 33 & $9(27.3 \%)$ & $24(72.7 \%)$ & \\
Surgical treatment & & & & \\
\hline PPI use & 210 & $68(32.4 \%)$ & $142(67.6 \%)$ & 0.06 \\
Yes & 13 & $1(7.7 \%)$ & $12(92.3 \%)$ & \\
No & & & & \\
\hline H2 antagonist use & 22 & $12(54.5 \%)$ & $10(45.5 \%)$ & 0.012 \\
Yes & 201 & $57(28.4 \%)$ & $144(71.6 \%)$ & \\
No & & & & 0.003 \\
\hline The use of antibiotics & 34 & $18(52.9 \%)$ & $16(47.1 \%)$ & \\
Yes & 189 & $51(27 \%)$ & $138(73 \%)$ & \\
\hline No & & & & \\
\hline Antacid use (calcium carbonate) & 34 & $14(41.2 \%)$ & $20(58.8 \%)$ & 0.2 \\
Yes & 189 & $55(29.1 \%)$ & $134(70.9 \%)$ & \\
No & & & & \\
\hline *chi-square test was used & & & & \\
\hline
\end{tabular}


Table 3

complication types of PUD

\begin{tabular}{|ll|}
\hline Type of complication & $\mathbf{n ( \% )}$ \\
\hline Gastrointestinal bleeding & $76(34.1 \%)$ \\
\hline Perforation & $12(5.4 \%)$ \\
\hline Penteration/fistula & $3(1.3 \%)$ \\
\hline Gastric outlet obstruction & $3(1.3 \%)$ \\
\hline Malignant transformation & $5(2.2 \%)$ \\
\hline No complications & $124(55.6 \%)$ \\
\hline
\end{tabular}

\section{Discussion:}

Peptic ulcer disease does not only put a huge financial burden on the healthcare sector but also its complications such as perforation can put patients at increased risk of morbidity and mortality. In our study several risk factors were assessed for association to 5-year recurrence rate and three risk factors were identified as statistically significant $\mathrm{p}$ value $<0.05$ which were H.pylori infection, NSAID, and steroid use. Our study found that H.pylori infection as a risk factor had 0.05 of 36 patients with H.pylori infection $16(44.4 \%)$ had recurrence, in contrast to patients not having H.pylori infection only 53 our 187 (28.3\%) had a recurrence. In accordance with our findings another study also found statistically significant difference in H.pylori infected patients vs non H.pylori infected patients in terms of recurrence where only 1 out of $9(11.1 \%)$ patients without H.pylori had a relapsed gastric ulcer within 1 year in contrast to 7 out of $18(38.9 \%)$ whom had an H.pylori had a relapsed peptic ulcer within 1 year [10]. They also found that H.pylori infection vs those not with H.pylori infection had not only increased risk of gastric ulcer recurrence, but also duodenal recurrence (0 vs $66.7 \%$ ) respectively. Another risk factor found to be associated with peptic ulcer recurrence in our study was NSAID use with a p value of 0.01 . 22 out of 49 (44.9\%) patients on NSAIDs had a recurrence of peptic ulcer, whereas only 47 out of 174 (27\%) patients not on NSAIDs had a recurrence. This result is similar to another study that found not only NSAID use had increased PUD recurrence but also increasing doses of NSAIDs had increased the risk of recurrence [11]. In their study total bare dose (CDDD) which is the total of allocated defined daily dose (DDD) were compared to those not taking NSAIDs which is defined as CDDD of 0 , they found that NSAID use vs nonuse had a matched cohort p-value of 0.004 . Nonsteroidal anti-inflammatory drugs (NSAIDs)-induced ulcers are normal in older patients, patients with various medications, patients with chronic conditions, Helicobacter pylori (H. pylori) infection, and background of peptic ulcers. A study recorded that 6.4 percent - 11.8 percent of patients who were administered NSAIDs developed peptic ulcer disease (PUD). Therefore, recurrence of peptic ulcer disease is strongly associated with the use of NSAIDs. Patients with PUD who have used NSAIDs upon early detection should then be screened and treated for $\mathrm{H}$. pylori infection [12]. 
In our study Steroid was found to be associated with peptic ulcer recurrence p value 0.009 . Out of 24 patients using steroids $13(54.2 \%)$ had recurrence of peptic ulcer, in contrast those not on steroids only 56 out of $199(28.1 \%)$ had a recurrence. Similarly, another study found that corticosteroids agents had an increased risk of peptic ulcer bleeding with odds ratio (OR) of 2.77 with $95 \%$ confidence interval of $0.62-$ 9.62 [13], they also found that immunosuppressive agents had the highest risk of peptic ulcer bleeding with odds ratios of and $95 \%$ confidence interval of $5.83 .88-22.88$.

Treatment plan and its association to peptic ulcer recurrence within 5 year was also assessed in our study and found to be statistically significant with $p$ value 0.02 . We found that avoiding risk factors had the lowest recurrence rate in contrast to other treatment plans such as eradication of H.pylori, acid suppression therapy, and surgical treatment $(6.7 \%, 50 \%, 30.3 \%, 27.3 \%$ respectively). This adds to the emphasis of risk factors identification in patients and addressing them. We also found that $\mathrm{H} 2$ antagonist use had a statistically significant impact on peptic ulcer recurrence with a p value of 0.012 .12 out of $22(54.5 \%)$ patients who were using $\mathrm{H} 2$ antagonists had a recurrence, where in contrast to those who did not receive $\mathrm{H} 2$ antagonists only 57 out of 201 (28.4\%) had a recurrence. This suggests a poor outcome in $\mathrm{H} 2$ antagonist therapy in treatment of peptic ulcer. In terms of PPI use and recurrence rate our study found that it was not statistically significant with a $p$ value of 0.06 . The use of antibiotics was statistically significant with a p-value of 0.003 . Patients who were on antibiotics had a higher incidence of recurrence vs those not on antibiotics $52.9 \%, 27 \%$ respectively. This suggests that while antibiotic therapy alone may eradicate H.pylori, it will also put patients at a higher risk of peptic ulcer recurrence in the future.

In terms of complications our study found that while $55.6 \%$ of patients faced no complications, the most common complication faced is gastrointestinal bleeding (GI bleed) $34.1 \%$ which is a life-threatening condition. There is also the issue of Idiopathic peptic ulcer disease (IPUD) which is the occurrence of peptic ulcers without specific causes, such as NSAIDs, H. pylori, and hypergastrinemia. The frequency of IPUD ranges from 1.3 percent to 27 percent due to differences in the rate of $\mathrm{H}$. pylori infection across various locations. The medical effects of IPUD are more critical than PUD due to H. pylori and NSAIDs, and the relapse rate is much higher. In addition, fatalities, and risk of repeated bleeding due to idiopathic bleeding ulcers are greater. In a study conducted recently the 5-year average reinfection rate of IPUD was stated to be 24.3 percent which is considerably higher than that for PUD-induced NSAIDs and eliminated $\mathrm{H}$. pylori-positive PUD. The lengthy relapse risk of PUD without $\mathrm{H}$. pylori due to effects of drugs is believed to be high; furthermore, there is currently insufficient evidence to support this concern. $\mathrm{H}$. pylori infection can be easily identified in PUD patients using several methods. And thus, it is critical to analyze the level of $\mathrm{H}$. pylori infection, the most powerful and reliable observable factor, as a significant predictor of PUD relapse. It is well known that infection with $\mathrm{H}$. pylori causes the recurrence of ulcers. [14].

Advanced medical therapy and innovative clinical endoscopic procedures have gradually reduced the need for surgical care in patients with PUD over the last 20 years. The most common endoscopic lesions following gastric surgery are marginal ulcers (MUs) occurring in the anastomotic region with a range of frequencies from 6 to 16 percent. The cause of MU is still unknown, although several aspects have been 
studied, such as the function of H. pylori infection, the significance of surgical anastomosis and other related risk factors (biliary reflux). Risk factors research that affects the incidence of complications in gastric stumps (cancers, ulcers) are of clinical concern, with a growing number of gastric operations in overweight or early gastric cancer patients. [15]. Our study aims to evaluate recurrence rate for PUD and analyze factors contributing to relapse. Complications of PUD and their impact was analyzed along with previous treatment regimen and their effects to determine efficacy of medications used in terms of recurrence.

This study has some limitations as it is a retrospective cross-sectional study and is a single center based which ultimately limits generalizability of results. The small sample size of 223 also limits the ability to show a difference between predictive values. Another limitation is the generalization of NSAIDs as different kinds of NSAIDs have various effects on PU, the same is true for steroids.

\section{Conclusion:}

Peptic ulcer disease places a significant burden on health care systems which advocate the need for effective management to prevent recurrence and complications. In this study three risk factors associated with 5-year peptic ulcer recurrence were found; $H$. Pylori, NSAID use, and steroid use. NSAIDs and steroids use are risk factors that are commonly prescribed for long term use. We found that simply avoiding risk factors had the lowest recurrence rate compared to $\mathrm{H}$. pylori eradication, acid suppression therapy, and surgical treatment. This emphasizes the need for identification of risk factors and avoidance for better long-term outcome. A multicenter prospective study is needed for effective management to prevent recurrence and complications of PUD.

\section{Declarations:}

\section{Declaration of Competing Interest}

The authors report no declarations of interest.

\section{Sources of funding}

None.

\section{Ethical Approval and Consent}

The ethical approval for the case report has been granted by the King Abdullah International Medical Research Centre with the reference number IRBC/1992/21. Informed written consent was obtained from all subjects and/or their legal guardian(s).

\section{Acknowledgment}

None 


\section{Author contribution}

$\mathrm{MH}$ : Consultant of Pediatric Gastroenterology who was responsible for the cases

YA: Leader, initiator, and supervisor of the case report.

MA: : writing the manuscript and proofing

MSA: writing the manuscript and proofing

ZMB: Date analysis

FB: writing the manuscript and data collecting

MA: writing the manuscript and data collecting

\section{Data availability}

All data generated or analyzed during this study available from the corresponding author on reasonable request

\section{References:}

1. Malik TF, Gnanapandithan K, Singh K. Peptic Ulcer Disease. [Updated 2021 Jul 29]. In: StatPearls [Internet]. Treasure Island (FL): StatPearls Publishing; 2021 Jan-. Available from: https://www.ncbi.nlm.nih.gov/books/NBK534792/

2. V. G. S. Kin Tong Chung, "Perforated peptic ulcer - an update," World journal of gastrointestinal surgery, pp. 1-12, 2017.

3. F. M. A. Fereydon Rezazadeh Zavoshti, "Therapeutics for Equine Gastric Ulcer Syndrome," The Veterinary clinics of North America. Equine practice., pp. 141-162, 2017.

4. K. M. R.,. E. M. Mechu Narayanan, "Peptic Ulcer Disease and Helicobacter pylori infection," Missouri Medicine, pp. 219-224, 2018.

5. J. H. Seo, "Long-Term Recurrence Rates of Peptic Ulcers without Helicobacter pylori," Gut and Liver, pp. 719-725, 2016.

6. S. J. H. C. H. C. H. C. M. D. Lau J.Y, "Systematic Review of the Epidemiology of Complicated Peptic Ulcer Disease: Incidence, Recurrence, Risk Factors and Mortality," Digestion, pp. 102-113, 2011.

7. P. C.-L. Y. A. M. M. C. S. Ángel Lanas, "Risk of Upper and Lower Gastrointestinal Bleeding in Patients Taking Nonsteroidal Anti-inflammatory Drugs, Antiplatelet Agents, or Anticoagulants," Clinical Gastroenterology and Hepatology, pp. 906-912, 2014.

8. Alqarni, Mohammed \& Bukhari, Ziad \& Alsinnari, Yaser \& Attar, Meshari \& Alzahrani, Abdulmalek \& Abukhodair, Abdulkarim \& Kadi, Ammar \& Alotibi, Maryam \& Jastaniah, Nisreen. (2021). 30-day 
mortality rate following hip fractures in elderly patients admitted to a tertiary care center. 14. 118122. 10.35841/1836-1935.14.4.118-122.

9. Attar, M., Alsinnari, Y. M., Alqarni, M. S., Bukhari, Z. M., Alzahrani, A., Abukhodair, A. W., Qadi, A., Alotibi, M., \& Jastaniah, N. A. (2021). Common Types of Falls in the Elderly Population, Their Associated Risk Factors and Prevention in a Tertiary Care Center. Cureus, 13(5), e14863. https://doi.org/10.7759/cureus.14863

10. Asaka M, Ohtaki T, Kato M, Kudo M, Kimura T, Meguro T, Horita S, Inoue K. Causal role of Helicobacter pylori in peptic ulcer relapse. J Gastroenterol. 1994 Jul;29 Suppl 7:134-8. PMID: 7921146.

11. Liang CM, Yang SC, Wu CK, et al. Risk of Recurrent Peptic Ulcer Disease in Patients Receiving Cumulative Defined Daily Dose of Nonsteroidal Anti-Inflammatory Drugs. J Clin Med. 2019;8(10):1722. Published 2019 Oct 18. doi:10.3390/jcm8101722

12. S.-C. Y. C.-K. W. Y.-C. L. W.-S. Y. W.-C. T. C.-H. L. Y.-H. Y. T.-H. T. C.-N. H. a. S.-K. C. Chih-Ming Liang, "Risk of Recurrent Peptic Ulcer Disease in Patients Receiving Cumulative Defined Daily Dose of Nonsteroidal Anti-Inflammatory Drugs," Journal of Clinical Medicine, p. 10, 2019.

13. Tomizawa, M., Shinozaki, F., Hasegawa, R., Shirai, Y., Motoyoshi, Y., Sugiyama, T. ... Ishige, N. (2017). Immunosuppressive agents are associated with peptic ulcer bleeding. Experimental and Therapeutic Medicine, 13, 1927-1931. https://doi.org/10.3892/etm.2017.4214

14. Seo JH, Hong SJ, Kim JH, et al. Long-Term Recurrence Rates of Peptic Ulcers without Helicobacter pylori. Gut Liver. 2016;10(5):719-725. doi:10.5009/gnl15262

15. A. N.,. C. B. S. B. R. N. S. M. a. M. I. Monica Pantea, "Factors Associated with Recurrent Ulcers in Patients with Gastric Surgery after More Than 15 Years: A Cross-Sectional Single-Center Study," Gastroenterology Research and Practice, p. N/A, 2018. 